\title{
METHODS OF CONFLICT PROBABILITY ESTIMATION AND DECISION MAKING FOR AIR TRAFFIC MANAGEMENT
}

\author{
V. Babak, V. Kharchenko, V. Vasylyev \\ National Aviation University, 1 Kosmonavta Komarova Ave., 03058, Kiev, Ukraine.E-mail: kharch@nau.edu.ua \\ Received 11042005 , accepted 06032006
}

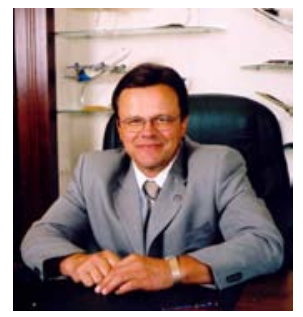

Vitaly Babak, Prof Dr Habil

Education: Kiev Polytechnic Institute.

Affiliations and functions: Rector of National Aviation University, Director of the Institute of Information and Diagnostic Systems, 1995 - Dr Sc (Eng.), Member of Academy of Sciences, Ukraine.

Areas of research: diagnostics of technological processes, laser and computer modeling and manufacturing, automatic diagnostics systems.

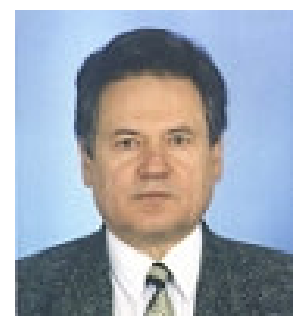

Volodymyr Kharchenko, Dr

Education: Kiev Institute of Civil Aviation Engineers.

Affiliations and functions: Vice-rector of National Aviation University on scientific work, Head of Aeronavigation Systems Department, 1994 - Dr Sc (Eng.).

Areas of research: The theory of situational analysis and control in socio-technical systems.

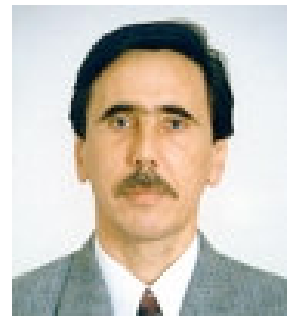

\author{
Volodymyr Vasylyev, Prof \\ Education: Kiev Institute of Civil Aviation Engineers. \\ Affiliations and functions: Professor of Aero navigation Systems Department at the National Aviation \\ University, 1983 - PhD (Eng.) \\ Areas of research: Systems theory, identification and prediction, decision support systems and its \\ application to the problems in air navigation and air traffic management.
}

\begin{abstract}
This research addresses the issue of conflict detection in Air Traffic Control (ATC) and in Airborne Separation Assurance System (ASAS) domains. Stochastic methods of conflict situation detection and conflict probability evaluation are presented. These methods can be used for air traffic conflict alert and avoidance systems for mid-range monitoring of air traffic and for flight safety. The mathematical formulation of the problem and the procedure of evaluation are presented. Two methods are introduced. One is based on fast statistical simulation of predicted violations of safe separation standards, and the other gives a closed-form analytic expression that can be applied to numerical evaluation methods. The next method proposed is a method of sequential evaluation of decision-making time limit to prevent a dangerous approach of the aircraft for short-range monitoring. The problem is solved by assuming that the estimation and prediction of trajectory are based on the spline-function method. The evaluation of the boundary instants for decision-making is achieved by solving the derived boundary equation for fixed decision-making distance. The distinguishing feature of this method is transformation of a confidence interval of predicted distance to a confidence interval of predicted time for estimation of the decision-making time limit.
\end{abstract}

Keywords: air traffic management, conflict detection, conflict probability, confidence interval, decision making.

\section{Introduction}

The principal and persistent requirement for Air Traffic Management (ATM) systems is provision of flight safety. That is why conflict detection and resolution play a key role, especially in the future ATM system, in which new concepts will allow aircraft to plan their enroute trajectories and resolve any conflicts with other aircraft in a distributed and co-operative manner.

In this paper, the research addresses the issue of conflict detection both in Air Traffic Control (ATC) and in Airborne Separation Assurance System (ASAS) 
domains. Conflict is defined as the predicted converging of aircraft in space and time, constituting a violation of a given set of separation minima.

It is known that mid-range conflict detection is used to provide centralized strategic information to the ATC for a probable conflict, whereas short-range conflict detection is performed on board and issues decentralized tactical instructions to pilots (and to ASAS) for a probable conflict within a time period of about five minutes.

Detection of potential conflict situation in mid-range monitoring is based on prediction of aircraft position uncertainty because of aircraft deviations from predicted trajectory. The most important sources of deviation are wind, aircraft dynamics, radar tracking, navigation and control errors, human errors, and system errors.

Stochastic methods of conflict detection are considered to be more flexible and promising than geometric methods [1].

The stochastic approach considered as a basic method is suggested in paper [4]. This approach aims to predict the probability of the separation between two aircraft falling below a certain threshold of separation. The proposed method puts some restrictions on the behaviour of aircraft, however. The authors make the following important assumptions: the predicted uncertainty in position is approximated as normally distributed, and the planned velocities and prediction errors of both aircraft are considered constant throughout the encounter.

The enhancement of this basic stochastic method has been proposed in paper [2]. This enhancement is made by introducing a dynamic dimension to the position error model and does not concern the method in fact.

The known collision risk approach is difficult for mathematical solution $[1,3]$. Collision risk equals the probability of the collision of two aircraft. The authors provide analytical expressions to estimate the probability of collision using other factors such as the incrossing rate and incrossing probability.

In this paper, stochastic methods of conflict situation detection and conflict probability evaluation are presented. These methods can be used for air traffic conflict alert and avoidance systems for mid-range monitoring of air traffic and to research flight safety. Two methods are introduced. One is based on fast statistical simulation of predicted violations of safe separation standards, and the other gives a closed-form analytic expression that can be applied to numerical evaluation methods.

Short-term path predictions typically do not use wind forecasts or flight plan data and are based on simple extrapolation of the current position of the aircraft and velocity vector. In short-range conflict detection, a potential conflict can be detected by state-based prediction methods. Thus, uncertainty of the future aircraft position depends mainly on the errors of trajectory measurement and prediction method.

The next method proposed is the method of sequential evaluation of the decision-making time limit to prevent a dangerous approach of the aircraft for short- range monitoring. The problem is solved by assuming that trajectory estimation and prediction are based on the spline-function method. The evaluation of the boundary instants for decision-making is achieved by solving the derived boundary equation for fixed decision-making distance. The distinguishing feature of this method is the transformation of a confidence interval of predicted distance to a confidence interval of predicted time for estimation of decision-making time limit.

\section{Methods of conflict probability estimation}

\subsection{Statement of problem}

Let us formulate a conflict detection problem for a two-aircraft encounter situation in a general form.

Let the position of each $j$-th aircraft $(j=\overline{1,2})$ in airspace at fixed moment $t$ be determined by the random vector $\mathbf{r}_{j}$, which has the Gaussian distribution $N\left(\mathbf{M}_{j}, \mathbf{D}_{j}\right)$ with mean vector $\mathbf{M}_{j} \in \mathfrak{R}^{3}$ and positive definite covariance matrix $\mathbf{D}_{j}$. It is assumed that vector $\mathbf{r}_{1}$ and vector $\mathbf{r}_{2}$ are independent.

Under the above assumptions, the distance vector between two aircraft $\mathbf{d}=\mathbf{r}_{1}-\mathbf{r}_{2}$ has the normal distribution $N(\mathbf{M}, \mathbf{D})$. This vector can be represented as the multi-dimensional random variable

$$
\mathbf{d}=\mathbf{M}+\mathbf{D} \gamma,
$$

where $\mathbf{M}=\mathbf{M}_{1}-\mathbf{M}_{2}$ is the mean vector; $\mathbf{D}=\sqrt{\mathbf{D}_{1}+\mathbf{D}_{2}}$ is a positive definite matrix; $\gamma$ is the vector of zero mean Gaussian random variables with unit variances, $N\left(0, \mathbf{I}_{3}\right) ; \mathbf{I}_{3}$ is unit $3 \times 3$ matrix.

The probability of violation of safe separation for the standard value of minimum separate distance $d_{\text {min }}$ between aircraft can be defined as

$$
P_{c}=P\left\{\left\|\mathbf{r}_{1}-\mathbf{r}_{2}\right\| \leq d_{\min }\right\},
$$

$\|\cdot\|$ denotes the Euclidean norm in $\Re^{3}$.

Taking into account (1), the expression (2) can be rewritten in the form

$$
P_{c}=P\left\{\|\mathbf{M}+\mathbf{D} \gamma\|^{2} \leq d_{\min }^{2}\right\}
$$

The decision about the potential conflict arising will be accepted if the conflict probability evaluated (3) becomes such that $P(t) \geq 1-\alpha$, where $\alpha$ is a significance level (usually $\alpha=0.05$ ).

\subsection{Stochastic fast simulation method of conflict probability estimation}

Let $\mathbf{e}_{1}, \mathbf{e}_{2}, \mathbf{e}_{3}$ be the orthonormal eigen-basis of matrix D . 

basis

Perform the expansion of mean vector $\mathbf{M}$ in this

$$
\mathbf{M}=q_{1} \mathbf{e}_{1}+q_{2} \mathbf{e}_{2}+q_{3} \mathbf{e}_{3},
$$

$q_{k}$ is defined by the scalar product $q_{k}=\left(\mathbf{M}, \mathbf{e}_{k}\right), k=\overline{1,3}$

Let us perform the representation on the basis of $\mathbf{e}_{1}, \mathbf{e}_{2}, \mathbf{e}_{3}$ :

$$
\mathbf{D} \gamma=\left[\begin{array}{c}
\lambda_{1} \gamma_{1} \\
\lambda_{2} \gamma_{2} \\
\lambda_{3} \gamma_{3}
\end{array}\right]
$$

where $\gamma_{1}, \gamma_{2}, \gamma_{3}$ are independent random variables with standard Gaussian probability distribution $N(0,1)$; $\lambda_{1}, \lambda_{2}, \lambda_{3}$ are the eigen-values of matrix $\mathbf{D}$.

Now we can represent the distance separation vector

$$
\mathbf{M}+\mathbf{D} \boldsymbol{\gamma}=\left[\begin{array}{c}
q_{1}+\lambda_{1} \gamma_{1} \\
q_{2}+\lambda_{2} \gamma_{2} \\
q_{3}+\lambda_{3} \gamma_{3}
\end{array}\right]
$$

and rewrite the Euclidean norm

$$
\begin{aligned}
& \|\mathbf{M}+\mathbf{D} \gamma\|^{2}=\zeta=\left(q_{1}+\lambda_{1} \gamma_{1}\right)^{2}+\left(q_{2}+\lambda_{2} \gamma_{2}\right)^{2}+\left(q_{3}+\lambda_{3} \gamma_{3}\right)^{2}= \\
& =\lambda_{1}^{2}\left(\frac{q_{1}}{\lambda_{1}}+\gamma_{1}\right)^{2}+\lambda_{2}^{2}\left(\frac{q_{2}}{\lambda_{2}}+\gamma_{2}\right)^{2}+\lambda_{3}^{2}\left(\frac{q_{3}}{\lambda_{3}}+\gamma_{3}\right)^{2},
\end{aligned}
$$

where $\zeta=\zeta\left(\lambda_{1}, \lambda_{2}, \lambda_{3}, q_{1}, q_{2}, q_{3}\right)$.

There is a program to simulate random variable $\zeta$ as a set of $n$ independent triple value of $\zeta_{1}, \ldots, \zeta_{n}$ from standard Gaussian random variables.

According to the strengthened law of large numbers

$$
\lim _{n \rightarrow \infty} \frac{1}{n} \sum_{i=1}^{n} I\left(\zeta_{i} \leq d_{\min }^{2}\right)=P\left\{\zeta \leq d_{\min }^{2}\right\},
$$

where $I$ is an indicator function of a random event.

When the value of $n$ is large, it is possible to consider that approximately

$$
P_{c}=\frac{1}{n} \sum_{i=1}^{n} I\left(\zeta_{i} \leq d_{\min }^{2}\right)=\frac{\#\left\{i: 1 \leq i \leq n, \zeta_{i} \leq d_{\min }^{2}\right\}}{n},
$$

where \# denotes an amount of points in a set for which a given condition is satisfied.

Thus if the value of $\mathbf{M}_{j}=\mathbf{M}_{j}(t)$ and $\mathbf{D}_{j}=\mathbf{D}_{j}(t)$ at fixed moment $t$ are known, it is possible to evaluate a conflict probability based on recorded independent random standard Gaussian extractions $\gamma_{1}^{1}, \ldots, \gamma_{1}^{n}$; $\gamma_{2}^{1}, \ldots, \gamma_{2}^{n} ; \gamma_{3}^{1}, \ldots \gamma_{3}^{n}$ by equation

$$
P_{c}=\frac{\#\left\{i: 1 \leq i \leq n,\left(q_{1}+\lambda_{1} \gamma_{1}^{i}\right)^{2}+\cdots+\left(q_{3}+\lambda_{3} \gamma_{3}^{i}\right)^{2} \leq d_{\min }^{2}\right\}}{n} .
$$

So instead of six sets of standard Gaussian variables, only three sets are needed.

The expressions considered concern instantaneous conflict probability at a fixed moment. A decision based on a single violation of the minimum separation distance can result in large level of false alarm. Thus there is an interest to evaluate the total probability of the encounter over a time of closest approach $t \in\left(t_{0}, t_{1}\right]$. We define the latter as

$$
\begin{aligned}
& P_{k}=\frac{1}{t_{k}-t_{0}} \int_{t_{0}}^{t_{k}} P(t) d t=\frac{1}{n\left(t_{k}-t_{0}\right)} \times \\
& \times \sum_{i=1}^{n} \int_{t_{0}}^{t_{k}} I\left(\left(q_{1}^{t}+\lambda_{1}^{t} \gamma_{1}^{i}\right)^{2}+\ldots+\left(q_{3}^{t}+\lambda_{3}^{t} \gamma_{3}^{i}\right)^{2} \leq d_{\text {min }}^{2}\right) d t .
\end{aligned}
$$

Using the Lebesque measure (mes) we can write the total probability in the form

$$
P_{k}=\frac{1}{n\left(t_{k}-t_{0}\right)} \sum_{i=1}^{n} \operatorname{mes}\left\{t \in\left(t_{0}, t_{k}\right]: f_{i}(t) \leq d_{\min }^{2}\right\},
$$

where $f_{i}(t)=\left(q_{1}^{t}+\lambda_{1}^{t} \gamma_{1}^{i}\right)^{2}+\cdots+\left(q_{3}^{t}+\lambda_{3}^{t} \gamma_{3}^{i}\right)^{2}$.

\subsection{Analytical method of conflict probability estimation}

The orthogonalization (4)-(6) enables the conflict probability to be derived in closed form.

Let's derive the analytical expression of conflict probability for a two dimensional case when two aircraft are flying at the same level.

After the expansion of a distance vector in an orthonormalized basis is performed, its components become independent. Then the distance vector $\mathbf{d}$ has Gaussian distribution $N\left(\left[q_{1}, q_{2}\right]^{T}, \operatorname{diag}\left[\lambda_{1}^{2}, \lambda_{2}^{2}\right]\right)$ and corresponding density function that can be noted as

$$
\begin{aligned}
& g(x, y)=g(x) g(y)= \\
& =\frac{1}{2 \pi \lambda_{1} \lambda_{2}} \exp \left(-\frac{1}{2}\left(\frac{\left(x-q_{1}\right)^{2}}{\lambda_{1}^{2}}+\frac{\left(y-q_{2}\right)^{2}}{\lambda_{2}^{2}}\right)\right) .
\end{aligned}
$$

In Figure 1 the dispersion ellipse relative to a point located according to mean values $q_{1}, q_{2}$ of variables $x$ and $y$ correspondingly is shown conditionally.

Conflict probability is defined as the probability of hitting the end of a random distance vector between aircraft $\mathbf{d}$ in a prohibited area bounded by a circle with radius $d_{\text {min }}$ centered in an origin of coordinates. 
The position of the end of the separation vector is determined by a mean vector $\left[q_{1}, q_{2}\right]^{T} \quad(5)$ and covariance matrix $\operatorname{diag}\left[\lambda_{1}^{2}, \lambda_{2}^{2}\right]$ (6).

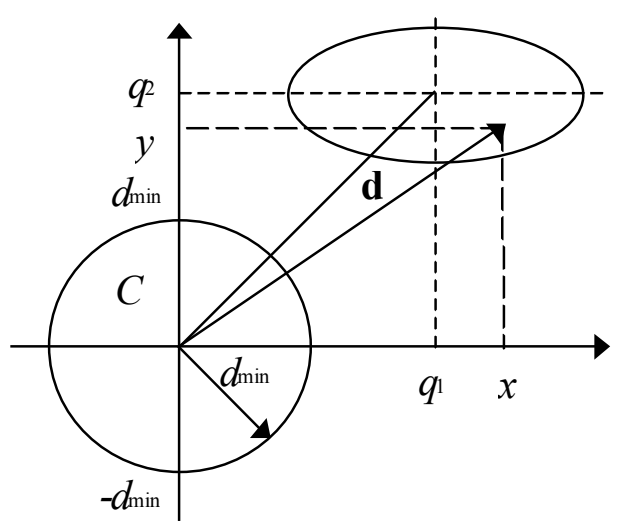

Fig 1. Geometric explanation of conflict probability evaluation

Thus conflict probability can be written under (7)

$$
\begin{aligned}
& P_{c}=P\{(x, y) \in C\}= \\
& =\iint_{C} \frac{1}{2 \pi \lambda_{1} \lambda_{2}} \exp \left(-\frac{1}{2}\left(\frac{\left(x-q_{1}\right)^{2}}{\lambda_{1}^{2}}+\frac{\left(y-q_{2}\right)^{2}}{\lambda_{2}^{2}}\right)\right) d x d y,
\end{aligned}
$$

where $C$ is a region of integration where the condition $x^{2}+y^{2} \leq d_{\min }^{2}$ is satisfied.

Considering the independence between random variables $x$ and $y$ the expression for conflict probability (8) can be noted as

$$
P_{c}=\iint_{C} \frac{1}{\lambda_{1} \sqrt{2 \pi}} e^{-\frac{\left(x-q_{1}\right)^{2}}{2 \lambda_{1}^{2}}} \frac{1}{\lambda_{2} \sqrt{2 \pi}} e^{-\frac{\left(y-q_{2}\right)^{2}}{2 \lambda_{2}^{2}}} d x d y .
$$

Integrating the external integral within the boundaries $-d_{\min }$ to $d_{\min }$, and the internal integral using the equation of a circle boundary $x^{2}+y^{2}=d_{\min }^{2}$ from $-\sqrt{d_{\min }^{2}-y^{2}}$ to $\sqrt{d_{\min }^{2}-y^{2}}$ define

$$
\begin{aligned}
& P_{c}=\int_{-d_{\min }}^{d_{\min }}\left(\frac{1}{\lambda_{2} \sqrt{2 \pi}} e^{-\frac{\left(y-q_{2}\right)^{2}}{2 \lambda_{2}^{2}}} \frac{1}{\lambda_{1} \sqrt{2 \pi}} \int_{-\sqrt{d_{\min }^{2}-y^{2}}}^{\sqrt{d_{\min }^{2}-y^{2}}} e^{-\frac{\left(x-q_{1}\right)^{2}}{2 \lambda_{1}^{2}}} d x\right) d y= \\
& =\frac{1}{\lambda_{2} \sqrt{2 \pi}} \int_{-d_{\min }}^{d_{\min }}\left(e ^ { - \frac { ( y - q _ { 2 } ) ^ { 2 } } { 2 \lambda _ { 2 } ^ { 2 } } } \left(\Phi\left(\frac{\sqrt{d_{\min }^{2}-y^{2}}-q_{1}}{\lambda_{1}}\right)-\right.\right. \\
& \left.\left.-\Phi\left(\frac{-\sqrt{d_{\min }^{2}-y^{2}}-q_{1}}{\lambda_{1}}\right)\right)\right) d y,
\end{aligned}
$$

where $\Phi(x)=\frac{1}{\sqrt{2 \pi}} \int_{-\infty}^{x} e^{-\frac{z^{2}}{2}} d z$

This analytical expression is applied to numerical evaluation methods.

\section{Sequential evaluation of decision making time limit}

\subsection{The logic of sequential time evaluation of decision making}

Because of uncertainties, the predicted conflict maybe will not take place in reality. Subsequently, deciding to move an aircraft in that case could sometimes be useless and could even generate other conflicts that would not occur had no manoeuvre been executed. On the other hand, excessive delay in decision-making can lead to the dangerous development of situation, time limitation, and infeasibility of optimal manoeuvring.

The basis of the proposed method consists of the following states.

Several levels of a decision-making (levels of warning) are installed depending on the air traffic situation and complication.

For each level:

- The rules and types of manoeuvres are specified

- The decision-making separation distances are calculated

- A set of physically feasible manoeuvres to avoid a potential conflict linked with the decision-making separation distance is calculated and specified.

Decision-making to avoid a conflict is made by a sequential prediction of the moments when aircraft will reach fixed distances of separation.

The flight time up to the predicted time limit of decision-making is calculated and displayed with information about feasible manoeuvres.

Essentially, it is the determination of a time limit for decision-making from a set of possible variants.

Considering trajectory prediction errors, the Decision-Making Time Limit Estimation should be done.

\subsection{Evaluation of the moment of passing the decision- making distance}

The objective is solved in several stages: estimating trajectory, extrapolating separation distance, and evaluating the moment that the decision-making distance has been passed.

Considering that for short-range conflict detection future aircraft position uncertainty depends mainly on the errors of trajectory measurement and prediction, the spline-function method is used.

We assume that there is information about aircraft position, speed, and heading, which allows 3D flight paths to be built.

Aircraft trajectory is determined as the vectorfunction of $\mathbf{r}=\mathbf{r}(t)$, all three components being cubic $C^{2}$-splines with the common elements $\tau_{0}<\tau_{1}<\ldots<\tau_{p}$. The use of cubic splines allows substantial geometric features of motion with using relatively small number of estimated trajectory parameters. The spline-function methods are realized in applicable algorithms as well. 
The spline $S=S(t)$, which is forming for one of the coordinate, for instance $y$, minimizes the functional

$$
\begin{aligned}
& Q(S)=\sum_{k=1}^{n} \frac{1}{\sigma_{y}^{2}}\left(y\left(t_{k}\right)-S\left(t_{k}\right)\right)^{2} \Delta t_{k}+ \\
& +\sum_{k=1}^{n} \frac{1}{\sigma_{v_{y}}^{2}}\left(v_{y}\left(t_{k}\right)-\frac{d S\left(t_{k}\right)}{d t}\right)^{2} \Delta t_{k}
\end{aligned}
$$

where $\sigma_{y}^{2}$ and $\sigma_{v_{y}}^{2}$ are dispersion of fluctuation errors of coordinate $y$ and speed $v_{y}$.

The optimization problem is parametrically solved by the expansion of $S(t)$ on $B$-splines.

The trajectory estimation is obtained by the formula

$$
\hat{\mathbf{r}}(t)=\sum_{i=1}^{p+3} \mathbf{B}_{i}(t) \hat{\mathbf{x}}_{i}
$$

where $\quad B_{i}(t), \quad 1 \leq i \leq p+3 \quad$ is normalized $B$-splines; $\hat{\mathbf{x}}_{i}=\left[\begin{array}{lll}\hat{x}_{i 1} & \hat{x_{i 2}} & \hat{x}_{i 3}\end{array}\right]^{T}$ is coefficients estimation of coordinate expansion [5].

Normalized spline is obtained from the expression

$$
B_{i}(t)=\left(\tau_{i}-\tau_{i-4}\right) \sum_{k=i-4}^{i} \frac{\left(\tau_{k}-t\right)_{+}^{3}}{d \omega_{i-4}\left(\tau_{k}\right) / d t}, \quad t \in\left[\tau_{0}, \tau_{p}\right],
$$

where

$$
\begin{aligned}
& \left(\tau_{k}-t\right)_{+}^{3}=\left\{\begin{array}{ccc}
\left(\tau_{k}-t\right)^{3}, & \text { if } t \leq \tau_{k} ; \\
0, & \text { if } t>\tau_{k} ;
\end{array}\right. \\
& \omega_{i}(t)=\prod_{j=i}^{i+4}\left(t-\tau_{j}\right) .
\end{aligned}
$$

The estimation of coefficients $\mathbf{x}=\left[x_{1}, \ldots, x_{p+3}\right]^{T}$, for instance for coordinate $y$, is defined as

$$
\hat{\mathbf{x}}=\mathbf{W}^{-1}\left[\mathbf{B}^{T} \boldsymbol{\Sigma}_{y}^{-1} \mathbf{S}_{t} \mathbf{y}^{*}+\dot{\mathbf{B}}^{T} \boldsymbol{\Sigma}_{v}^{-1} \mathbf{S}_{t} \mathbf{v}_{y}^{*}\right]
$$

where $\mathbf{B}=\left[b_{k i}\right],(1 \leq k \leq n, 1 \leq i \leq p+3)$ is the matrix consisting of elements $b_{k i}=B_{i}\left(t_{k}\right)$

$$
\mathbf{W}=\mathbf{B}^{T} \boldsymbol{\Sigma}_{y}^{-1} \mathbf{S}_{t} \mathbf{B}+\dot{\mathbf{B}}^{T} \boldsymbol{\Sigma}_{v}^{-1} \mathbf{S}_{t} \dot{\mathbf{B}} ; \quad \dot{\mathbf{B}}=\frac{d \mathbf{B}}{d t} ;
$$

$\mathbf{y}^{*}, \mathbf{v}_{y}^{*}$ are the measurements of coordinate and speed with mean square errors $\sigma_{y}, \sigma_{v_{y}}$ correspondingly;

$$
\begin{gathered}
\mathbf{S}_{t}=\operatorname{diag}\left[\Delta t_{1}, \ldots, \Delta t_{n}\right] ; \\
\boldsymbol{\Sigma}_{y}=\operatorname{diag}\left[\sigma_{y}^{2}\left(t_{1}\right), \ldots, \sigma_{y}^{2}\left(t_{n}\right)\right] ; \\
\boldsymbol{\Sigma}_{v}=\operatorname{diag}\left[\sigma_{v_{y}}^{2}\left(t_{1}\right), \ldots, \sigma_{v_{y}}^{2}\left(t_{n}\right)\right] ;
\end{gathered}
$$

The estimation of coordinate $y$ under (9), (10) is defined as $\hat{y}(t)=\sum_{i=1}^{p+3} B_{i}(t) \hat{\mathbf{x}}_{i}$,

here $\mathbf{x}=\left[x_{1}, \ldots, x_{p+3}\right]^{T}$.

Then after the last measurement at time $t_{n}$, the flight path (11) can be extrapolated. Extrapolation is obtained on interval $\left[t_{n}, \tau_{p}\right)$ where cubic curves on interval $\left[\tau_{p-1}, t_{n}\right]$ are preserved.

Having predicted spline trajectory for each aircraft, the predicted estimation of a separate distance between two aircraft at a fixed moment $t$ can be defined from the expression

$$
\hat{d}(t)=\sqrt{\sum_{k=1}^{3}\left[\mathbf{b}_{k, 1}^{T}(t) \hat{\mathbf{z}}_{k, 1}-\mathbf{b}_{k, 2}^{T}(t) \hat{\mathbf{z}}_{k, 2}\right]^{2}},
$$

where $k=\overline{1,3}$ is an index defining one of the coordinates; a second index $j=\overline{1,2}$ marks the components related with one of two aircraft; $\mathbf{b}_{k}(t)=\left[B_{i}\right]_{i=p}^{p+3}$ is the vector of $B$-splines values; $\quad \mathbf{z}_{k}=\left[x_{i k}\right]_{i=p}^{p+3}$ is the vector of coefficients of coordinate expansion on basic splines.

Using expression (12) for the fixed decision-making distance $d_{s}$, the appropriate moment $t_{s}$ of passing this distance can be evaluated.

\subsection{Evaluation of decision-making time limit}

Taking into account the prediction errors carries out the evaluation of the decision-making time limit. Such evaluation is made by transition from confidence position interval to confidence time interval.

At first the confidence distance interval $\Delta d(t)=|d(t)-\hat{d}(t)|$ with probability of not less than $(1-\alpha)$ is evaluated. Considering spline extrapolation and Gaussian distribution of measurement errors, the confidence distance interval is defined as

$$
\Delta d(t) \leq u(t) \sqrt{\left(\chi_{24}^{2}\right)_{\alpha}}
$$

where $\left(\chi_{24}^{2}\right)_{\alpha}$ is $\alpha$-quantile of $\chi_{24}^{2}$ distribution; $P\left\{\chi_{24}^{2}>\left(\chi_{24}^{2}\right)_{\alpha}\right\}=\alpha$

$$
u(t)=\sqrt{\sum_{k=1}^{3} \sum_{j=1}^{2}\left(\mathbf{D}_{k, j} \mathbf{b}_{k, j}(t), \mathbf{b}_{k, j}(t)\right)}
$$

$\mathbf{D}_{k}$ is a covariance matrix of vector $\hat{\mathbf{z}}_{k} ; j$ is aircraft index; $(\cdot, \cdot)$ denotes a scalar product. 
This confidence interval depends on covariance matrix $\mathbf{D}_{k}$, and when time is varying the equation (13) gives the confidence band

$$
\hat{d}(t)-\Delta d \leq d(t) \leq \hat{d}(t)+\Delta d
$$

The boundaries of the confidence distance interval are defined by the equation

$$
d(t)=\hat{d}(t) \mp u(t) \sqrt{\left(\chi_{24}^{2}\right)_{\alpha}} .
$$

The evaluation of the decision-making time limit is made by solving the boundary equation (14) for fixed decision-making distance $d_{s}$ and corresponding passing time $t_{s}$.

The confidence time interval is writing down by the inequality

$$
t_{s}-\Delta T^{\prime} \leq t \leq t_{s}+\Delta T^{\prime \prime}
$$

Note that in common case $\Delta T^{\prime} \neq \Delta T^{\prime \prime}$.

Thus the decision-making time limit is equal

$$
t_{\lim }=t^{\prime}=t_{s}-\Delta T^{\prime} \text {. }
$$

The procedure described for time interval $t \in\left[t_{1}, t_{2}\right]$ is illustrated in Figure 2. Dotted lines are the boundary of the confidence interval for distance.

The iterative algorithm to search the decisionmaking time limit is proposed as well:

$$
\begin{aligned}
& d_{\mathrm{s}}=\hat{d}\left(t_{s}\right) \\
& \tau=0 ;
\end{aligned}
$$

While $\left(\hat{d}\left(t_{s}-\tau\right)-\Delta d\left(t_{s}-\tau\right)\right)<d_{\mathrm{s}}$ do

$$
\tau=\tau+\Delta \tau
$$

\section{end}

$$
t_{\lim }=t_{s}-\tau \text {. }
$$

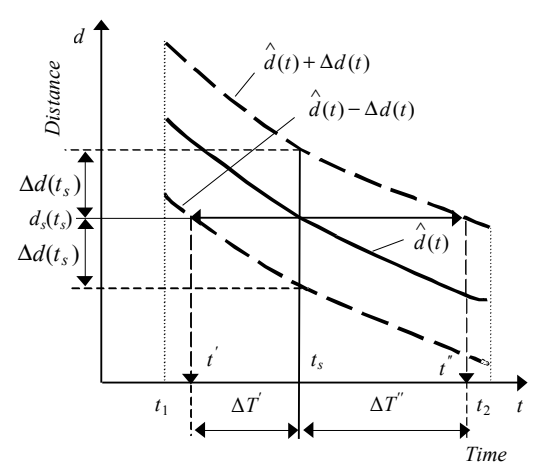

Fig 2. Transformation of the confidence distance interval to the confidence time interval

\section{Conclusions}

For mid-range monitoring of a conflict situation the orthogonalization procedure introduced provides a fast statistical simulation of predicted violations of safe separation standards and estimation of conflict probability by minimizing sample size of standard random values.

The analytical expression for conflict probability estimation is derived in closed-form. It is applied to numerical evaluation methods and can provide precise conflict estimation.

For short-range monitoring of a conflict, the method introduced consists of determining decision-making levels and sequential evaluation of decision-making time limit for choice of maneuver type to avoid a potential conflict.

The decision-making time limit is evaluated by taking into account the errors in the trajectory extrapolation method.

A distinguishing feature of this method is the transformation of the predicted confidence position interval to the confidence time interval for the estimation of the decision-making time limit.

The stochastic methods presented can be used both in Airborne Separation Assurance System and in the Conflict Alert algorithm in ATC and also for research of flight safety, including computer simulation.

\section{References}

1. Bakker G.J., Kremer H.J., Blom H.A.P. Probabilistic Approaches Toward Conflict Prediction // Air Transportation Systems Engineering, AIAA. 2001. - P. 677-694.

2. Blin K., Bonnans F., Hoffman E., Zeghal K. A Stochastic Conflict Detection Method Integrating Planned Heading and Velocity Changes // IEEE Conference on Decision and Control, Sydney, December 2000. - Sydney, 2000.

3. Blom H.A.P., Bakker G.J., Blanker P.J.G. at al. Accident Risk Assessment for Advanced ATM // Air Transportation Systems Engineering / Ed. G.L. Donohue and A.G. Zellweger. - AIAA, 2001. - P. 463-480.

4. Paielli R.A., Erzberger H. Conflict Probability Estimation for Free Flight // Journal of Guidance, Control and Dynamics. - 1997. - Vol. 20, № 3. - P. 588-596.

5. Харченко В.П., Кукуш О.Г., Бабак Є.А. и др. Класифікація конфліктних ситуацій між літальними апаратами та вибір зон безпеки. // Вiсн. НАУ. - 2002. - №3. - C. 79-88 (in Ukrainian).

\section{List of symbols}

$$
\begin{array}{ll}
\mathbf{r} & - \text { vector of aircraft trajectory } \\
\mathbf{M} & - \text { mean vector } \\
\mathbf{D} & - \text { covariance matrix } \\
\mathfrak{R}^{3} & - \text { three-dimensional space }
\end{array}
$$


d - distance vector between two aircraft

$\gamma \quad-$ standard Gaussian random variable

I $\quad-$ unit matrix

$d_{\text {min }} \quad-$ safe separation standard

$P_{c} \quad-$ conflict probability

$P_{k} \quad-$ total conflict probability over defined time

$\|\cdot\| \quad-$ denotes Euclidean norm

$\wedge \quad-$ denotes an estimation

$(\cdot, \cdot) \quad-$ denotes a scalar product

mes - denotes Lebesque measure

$\mathbf{e}_{1}, \mathbf{e}_{2}, \mathbf{e}_{3}$ - orthonormal basis vectors

I $\quad$ - indicator function ( $I=1$ if defined condition

is satisfied, $I=0$ otherwise).

\# - amount of points in a set for which defined

condition is satisfied

$C \quad-$ region of a conflict

$S \quad-$ spline

$Q(S) \quad-$ functional

$\tau_{0}<\tau_{1}<\ldots<\tau_{p}-$ common spline elements

$t \quad-$ time

$\Delta t \quad-$ time discretization step

$t_{k} \quad-$ fixed moment of time

$t_{n} \quad-$ time of last measurement

$t_{s} \quad-$ moment of passing the fixed decision-

making distance

$t_{\lim } \quad-$ decision-making time limit

$y \quad-$ one of the coordinates of aircraft trajectory

$\mathbf{y}^{*} \quad-$ vector of measurements of the coordinate $y$

$v_{y} \quad-$ aircraft speed along the coordinate $y$

$\mathbf{v}_{y}^{*} \quad-$ vector of measurements of aircraft speed

along the coordinate $y$

$\sigma_{y} \quad-$ mean square error of measurements of the

coordinate $y$

$\sigma_{v_{y}} \quad-$ mean square error of measurements of

aircraft speed $v_{y}$

$B \quad-$ normalized $B$-spline

$\mathbf{B}=\left[b_{k i}\right]-$ matrix consisting of normalized splines

$b_{k i}=B_{i}\left(t_{k}\right)$ for all moments $t_{k}$

$\mathbf{b}(t) \quad-$ vector of predicting $B$-splines values at a

fixed moment $t$ for one of the coordinates

$\mathbf{x}_{i} \quad-$ vector of coefficients of coordinate

expansion on basic splines.

$\mathbf{x}-$ vector of coefficients of coordinate

expansion on basic splines for one of the coordinates

$\mathbf{z}-$ vector of coefficients of coordinate

expansion on predicting basic splines for one of the coordinates

$\mathbf{S}_{t} \quad$ - diagonal matrix consisting of time

discretization steps
$\Sigma_{y} \quad$ - diagonal matrix consisting of error

dispersion of measurements of coordinate $y$.

$\boldsymbol{\Sigma}_{v} \quad$ - diagonal matrix consisting of error

dispersion of measurements of speed $v_{y}$.

$\left(\chi_{24}^{2}\right)_{\alpha}-\alpha$-quantile of $\chi_{24}^{2}$ distribution

$d_{s} \quad-$ fixed decision-making distance

$\Delta d \quad-$ confidence distance interval

$\Delta T \quad-$ confidence time interval 